\title{
Meta
}

Journal des traducteurs

Translators' Journal

\section{Les mammifères aquatiques : lexique anglais-français des mammifères marins actuels ou récemment disparus, y compris les espèces d'eau douce des groupes surtout marins}

\section{Vladimir N. Nekrassoff}

Volume 25, numéro 3, septembre 1980

URI : https://id.erudit.org/iderudit/003718ar

DOI : https://doi.org/10.7202/003718ar

Aller au sommaire du numéro

Éditeur(s)

Les Presses de l'Université de Montréal

ISSN

0026-0452 (imprimé)

1492-1421 (numérique)

Découvrir la revue

Citer cet article

Nekrassoff, V. N. (1980). Les mammifères aquatiques : lexique anglais-français des mammifères marins actuels ou récemment disparus, y compris les espèces d'eau douce des groupes surtout marins. Meta, 25(3), 325-345.

https://doi.org/10.7202/003718ar d'utilisation que vous pouvez consulter en ligne. 


\section{PROBLÈMES ET \\ SOLUTIONS}

\section{LES MAMMIFÈRES AQUATIQUES: LEXIQUE ANGLAIS-FRANÇAIS DES MAMMIFÈRES MARINS ACTUELS OU RÉCEMMENT DISPARUS, Y COMPRIS LES ESPĖCES D'EAU DOUCE DES GROUPES SURTOUT MARINS*}

Le présent lexique se fonde sur des études et révisions taxinomiques récentes. Le nom scientifique est suivi des noms courants anglais et français. Les index comportent d'autres noms courants et renvoient au lexique même.

Les noms scientifiques sont particulièrement importants puisque, malgré les différences d'appellation d'un pays à l'autre, le nom scientifique reste le même. Les synonymes présentés dans le lexique reflètent les révisions apportées par des zoologistes qui ont remarqué que plusieurs mammifères marins avaient été décrits plus d'une fois sous différents noms scientifiques.

La classification des mammifères marins est difficile parce que ces animaux sont peu connus. Les uns vivent en haute mer; d'autres habitent des îles océaniques isolées ou des champs de glaces polaires. Quelques siréniens et cétacés plus petits habitent des eaux tropicales rarement visitées par les mammalogistes. Leur étude est à la fois difficile et dispendieuse. Toute liste de mammifères marins, y compris le présent lexique, doit donc être considérée comme provisoire.

Mitchell et Tedford (1973) ont étudié en détail le développement phylogénique des pinnipèdes et ont montré qu'ils avaient les mêmes ancêtres que les mustélidés (belettes), les procyonidés (ratons laveurs), les canidés (chiens) et les ursidés (ours), qui forment ensemble le sous-ordre des fissipèdes, une des deux grandes divisions des carnivores actuels. Rice (dans Anderson et Jones, 1967), suivant Kleinenberg (1958), a distingué les cétacés qui ont des dents (odontocètes) des cétacés munis de fanons (mysticètes) et il en a fait deux ordres distincts. Pour ce qui est des odontocètes, les espèces des genres Platanista, Sousa, Sotalia et Tursiops ont été modifiées en fonction des recommandations du comité scientifique de l'International Whaling Commission (1975, "List of * Une version allemande du présent lexique a été publiée dans Lebende Sprachen (Langenscheidt,
Munich), vol. 25(2), 1980 . 
Smaller Cetaceans Recognized"). La synonymie desı pinnipèdes se fonde sur Scheffer (1958), celle des cétacés sur Hershkovitz (1966).

Le présent lexique ne tient compte que des mammifères marins récents, les formes fossiles étant signalées dans Piveteau $(1958,1961)$ (en français), Reinhart (1959) (en anglais), Matthes (1962) (en allemand), King (1964) (en anglais) et Romer (1966) (en anglais).

1. Arctocephalus

2. Arctocephalus australis

3. Arctocephalus australis australis

Arctocephalus doriferus = Arctocephalus pusillus doriferus

Arctocephalus elegans = Arctocephalus tropicalis

4. Arctocephalus forsteri Arctocephalus forsteri doriferus $=A$. pusillus doriferus

5. Arctocephalus galopagoensis

6. Arctocephalus townsendi

7. Arctocephalus gazella

8. Arctocephalus philippii

9. Arctocephalus pusillus

10. Arctocephalus pusillus doriferus

11. Arctocephalus pusillus pusillus

Arctocephalus tasmanicus =

A. pusillus doriferus

12. Arctocephalus tropicalis

Arctocephalus ursinus $=$ Callorhinus ursinus

Arctophoca $=$ Arctocephalus

Balaena australis = Balaena glacialis

Balaena biscayensis $=\mathbf{B}$. glacialis

13. Balaena glacialis

14. Balaena glacialis australis

15. Balaena glacialis glacialis

Balaena japonica $=\mathrm{B}$. glacialis

16. Balaena mysticetus

Balaena sieboldii $=\mathbf{B}$. glacialis

17. Balaenidae

18. Balaenoptera southern fur seals

South American fur seal

Falkland fur seal

Antipodean fur seal

Galapagos fur seal

Guadalupe fur seal

Antarctic fur seal

Juan Fernandez fur seal

giant fur seal

Victorian fur seal

South African fur seal

Subantarctic fur seal

right whale

southern right whale

North American right whale

bowhead whale

bowhead whales

rorquals otaries à fourrure du sud

otarie à fourrure australe

otarie des Falkland

otarie d'Australie

otarie de Galapagos

otarie à fourrure de Townsend

otarie de Kerguelen

otarie de Chile

otarie à fourrure géante

otarie du sud de l'Australie

otarie à fourrure d'Afrique du Sud

otarie à fourrure des îles Kerguelen

baleine noire

baleine noire du Sud

baleine noire de l'Atlantique Nord

baleine boréale (F.)

baleine franche (Can.)

baleines

rorquals 
19. Balaenoptera acutorostrata

20. Balaenoptera acutorostrata acutorostrata

21. Balaenoptera acutorostrata bonaerensis

22. Balaenoptera acutorostrata davidsoni

Balaenoptera bonaerensis $=$ Balaenoptera acutorostrata

23. Balaenoptera borealis

24. Balaenoptera borealis borealis

25. Balaenoptera borealis schlegellii

Balaenoptera brydei $=\mathbf{B}$. eden

Balaenoptera davidsoni $=\mathrm{B}$. acutorostrata

26. Balaenoptera edeni

Balaenoptera huttoni $=\mathrm{B}$. acutorostrata

27. Balaenoptera musculus

28. Balaenoptera musculus brevicauda

29. Balaenoptera musculus intermedius

30. Balaenoptera musculus musculus

31. Balaenoptera physalus

32. Balaenoptera physalus quoyi

33. Balaenoptera physalus physalus

34. Balaenopteridae

Balenidae $=$ Balaenidae

35. Berardius

36. Berardius arnouxii

Berardius arnuxi $=\mathbf{B}$. arnouxii

37. Berardius bairdii

Callorhinus alascana $=\mathrm{C}$.

alascensis

Callorhinus alascensis $=\mathrm{C}$. ursinus

Callorhinus curilensis $=\mathrm{C}$.

ursinus

Callorhinus cynocephalus $=$

C. ursinus

Callorhinus mimicus $=\mathrm{C}$. ursinus

38. Callorhinus ursinus Calorhinus = Callorhinus minke whale

North Atlantic minke

whale

souther hemisphere

minke whale

North Pacific minke whale

Sei whale

northern hemisphere Sei

whale

southern hemisphere Sei

whale

Bryde's whale

blue whale

southern Indian Ocean

blue whale

Antarctic blue whale

North Atlantic blue whale whale

fin whale

southern hemisphere fin

whale

northern hemisphere fin

whale

rorquals (or fin whales)

giant bottle-nosed whales

southern giant bottlenose

whale

North Pacific giant

bottlenose whale

northern fur seal petit rorqual

petit rorqual de l'Atlantique

Nord

petit rorqual de

I'hémisphère sud

petit rorqual du Pacifique Nord

rorqual boréal

rorqual boréal de l'hémisphère sud

rorqual boréal de l'hémisphère

balénoptère boréal de Bryde

rorqual bleu

petit rorqual bleu

rorqual bleu de l'Antarctique

rorqual bleu de l'Atlantique Nord

rorqual commun

rorqual commun de

l'hémisphère sud

rorqual commun de

l'hémisphère nord

rorquals

baleines noires

baleine d'Amoux

grande baleine-à-bec 
39. Caperea marginata

40. Carnivora

Cephalorhynchinae $=$ Cephalor chynchus

41. Cephalorhynchus

Cephalorhynchus albifrons $=$

C. hectori

Cephalorhynchus albiventris $=$ C. eutropia

42. Cephalorhynchus commersonii piebald dolphin

43. Cephalorhynchus eutropia black dolphin

44. Cephalorhynchus heavisidei Heaviside's dolphin

45. Cephalorhynchus hectori

46. Cetacea

47. Choeropsis liberiensis Cogia $=$ Kogia

48. Cystophora cristata

49. Cystophorinae

Delphinapterus dorofeevi $=$

D. leucas

Delphinapterus friemani $=\mathrm{D}$. leucas

Delphinapterus kingii $=\mathrm{D}$.

leucas

50. Delphinapterus leucas

51. Delphinapterus leucas dorofeevi

52. Delphinidae

53. Delphininae Delphinus bairdii $=\mathrm{D}$. delphis

54. Delphinus boryi Delphinus capensis $=\mathrm{D}$. delphis

55. Delphinus delphis

56. Delphinus delphis tropicalis

Delphinus dussumieri $=\mathrm{D}$. delphis

Delphinus longirostris $=\mathrm{D}$. delphis

Delphinus roseiventris $=$ Stenella longirostris

Delphinus tropicalis = D. delphis

Dugongidae $=$ Halicoridae Electra $=$ Peponocephala

57. Enhydra lutris

58. Enhydra lutris gracilis

59. Enhydra lutris lutris

60. Enhydra lutris nereis pygmy right whale

carnivores (flesh-eaters)

Commerson's dolphins pied dolphin

whales, dolphins, and porpoises

pigmy hippopotamus

hooded seal

crested seals and sea

elephants

beluga

Okhotsk Sea beluga

dolphins and porpoises

true dolphins

Bory's dolphin

saddleback dolphin

Arabian Sea saddleback dolphin sea otter

Kamchatka sea otter northern sea otter southern sea otter baleine pygmée

carnivores

dauphins de Commerson

dauphin de Commerson

dauphin noir

dauphin de Heaviside

dauphin de Hector

cétacés

hippopotame nain

phoque à capuchon

cystophorinés béluga (vrai)

béluga de la mer d'Okhotsk

delphinidés (dauphins)

dauphins vrais

dauphin commun

dauphin commun de la mer d'Oman loutre de mer

loutre de mer de Kamchatka loutre de mer septentrionale loutre de mer australe 
61. Erignathus barbatus

62. Erignathus barbatus barbatus

63. Erignathus barbatus nauticus

64. Eschrichtiidae

Eschrichtius gibbosus $=\mathrm{E}$. robustus

Eschrichtius glaucus $=\mathrm{E}$. robustus

65. Eschrichtius robustus Eubalaena $=$ Balaena

66. Eumetopias

67. Eumetopias jubatus Eumetopias stellerii $=\mathrm{E}$. jubatus

68. Feresa attenuata Feresa intermedia $=\mathrm{F}$. attenuata Feresa occulta $=F$. attenuata

69. Globicephala

Globicephala brachyptera $=\mathrm{B}$. macrorhynchus

Globicephala edwardii $=\mathbf{G}$. melaena

Globicephala leucosagmaphora $=\mathrm{G}$. melaena

70. Globicephala macrorhyncha

71. Globicephala melaena

72. Globicephala melaena edwardii

Globicephalus melas $=\mathrm{G}$.

melaena

Globicephala scammonii $=\mathrm{G}$. macrorhynchus

73. Globicephala sieboldii Grampidelphis $=$ Grampus

74. Grampus griseus

Pacific pilot whale

whitehead grampus

Grampus orca $=$ Orcinus orca

Grampus rectipinna $=$ Orcinus orca

Gypsophoca $=$ Arctocephalus

75. Halichoerus grypus

76. Halicoridae

77. Hippopotamus amphibius Histriophoca $=$ Phoca

78. Hydrodamalis gigas Hydrodamalis stelleri $=H$. gigas

79. Hydrurga leptonyx

80. Hyperoodon

81. Hyperoodon ampullatus bearded seal

Atlantic bearded seal

Pacific bearded seal grey whales

grey whale

sea lions

northern sea lion

pygmy killer whale

pilot whales

shortfin pilot whale longfin pilot whale

southern hemisphere longfin pilot whale grey seal

dugongs

hippopotamus

Steller's sea-cow

leopard seal

bottle-nosed whales

North Atlantic bottlenose whale

phoque à barbe (F.)

phoque barbu de l'Atlantique

phoque barbu du Pacifique

eschrichtiides

baleine grise (de Californie)

lions de mer

otarie de Steller

orque naine (F.)

globicéphales (F.)

globicéphale du Pacifique globicéphale noir de l'Atlantique (Can.)

globicéphale noir de l'hémisphère sud

globicéphale de Siebold

dauphin gris

phoque gris

dugongidés

hippopotame

rhytine de Steller

phoque léopard

baleines-à-bec

baleine-à-bec commune 
82. Hyperoodon planifrons Hyperoodon rostratus $=\mathrm{H}$. ampullatus

83. Indopacetus pacificus

84. Inia geoffrensis

85. Iniidae

86. Kogia breviceps

86a. Kogia simus

87. Lagenodelphis hosei

88. Lagenorhynchus acutus

89. Lagenorhynchus albirostris

90. Lagenorhynchus australis

91. Lagenorhynchus cruciger Lagenorhynchus electra $=$ Peponocephala electra

Lagenorhynchus fitzroyi $=\mathrm{L}$. obscurus

92. Lagenorhynchus obliquidens

93. Lagenorhynchus obscurus Lagenorhynchus ognevi $=\mathrm{L}$. obliquidens

Lagenorhynchus superciliosus $=$ L. obscurus

94. Lagenorhynchus thicoloea Lagenorhynchus wilsoni $=\mathrm{L}$. cruciger

95. Leptonychotes weddelli

96. Lipotes vexillifer

97. Lissodelphinae

98. Lissodelphis borealis

99. Lissodelphis borealis albiventris

100. Lissodelphis peronii

101. Lobodon carcinophagus

102. Lobodontinae

103. Lutra felina

104. Lutra lutra Megaptera boops $=\mathrm{M}$. novaeangliae

Megaptera longinmana $=$ $M$. novaeangliae

Megaptera nodosa $=\mathbf{M}$. novaeangliae

105. Megaptera novaeangliae

106. Mesoplodon

107. Mesoplodon bidens

108. Mesoplodon bowdoini

109. Mesoplodon carlhubbsi flathead bottlenose whale

Indo-Pacific beaked whale Amazon dolphin

Amazon dolphins

pygmy sperm whale

dwarf sperm whale

shortsnouted whitebelly

dolphin

Atlantic whiteside dolphin

whitebeak dolphin

blackchin dolphin

hourglass dolphin

Pacific whitesided dolphin

dusky dolphin

Gray's white-sided dolphin

Weddell seal

whiteflag dolphin

right whale dolphins

northern right-whale dolphin

Japanese northern rightwhale dolphin

southern right-whale dolphin crab-eater seal

southern seals

chungungo

Eurasian otter

humpback whale

beaked whales (or cowfish)

North Sea beaked whale

deepcrest whale

archbeak whale hypéroodon du sud

baleine-à-bec de Longman

inie de Geoffroy

iniidés

cachalot pygmée

dauphin de Bornéo

dauphin-à flancs blancs

dauphin-à-bec blanc

dauphin-à-bec de Peale

dauphin à flancs blancs du Pacifique

dauphin de Fitzroy

\section{phoque de Weddell}

dauphin d'eau douce de Chine dauphins-baleines francs

dauphin à dos lisse

dauphin à dos lisse du Japon

dauphin de Peron

phoque crabier

phoques du sud

loutre eurasienne

rorqual à bosse (Can.)

mésoplodons

baleine-à-bec de Sowerby

baleine-à-bec de Moore 
110. Mesoplodon densirostris

111. Mesoplodon europaeus Mesoplodon gervaisi $=\mathbf{M}$. europaeus

112. Mesoplodon ginkgodens

113. Mesoplodon grayi

114. Mesoplodon hectori

Mesoplodon hotaula $=\mathrm{M}$ gingkodens

115. Mesoplodon layardii

116. Mesoplodon mirus

Mesoplodon pacificus = Indopacetus pacificus

117. Mesoplodon stejnegeri

118. Mirounga

119. Mirounga angustirostris

120. Mirounga leonina

121. Monachinae

Monachus albiventer $=\mathbf{M}$. monachus

122. Monachus monachus

123. Monachus schauinsland

124. Monachus tropicalis

125. Monodon monocerus

126. Monodontidae

127. Monodontoidea Mystacoceti $=$ Mysticeti

128. Mysticeti

129. Nasalis larvatus

Neobalaena $=$ Caperea

Neomeris = Neophocaena

130. Neophoca cinerea

Neophoca hookeri $=$

Phocarctos hookeri

Neophoca lobatus $=$ N. cinerea

131. Neophocaena asiaeorientalis ${ }^{1}$ finless porpoise from China

132. Neophocaena phocaenoides ${ }^{1}$

133. Neophocaena phocaenoides ${ }^{1}$ asiaeorientalis

134. Neophocaena sunameri ${ }^{1}$

Nodus $=$ Mesoplodon finless porpoise (from Pakistan to Borneo)

finless porpoise from

China and Japan

densebeak whale

Antillean beaked whale

ginkgo-tooth whale

scamperdown whale

New Zealand beaked whale

straptooth whale

True's beaked whale

sabertooth whale

elephant seals

northern elephant seal

southern elephant seal

monk seals

monk seal (Mediterranean)

Hawaiian monk seal

West Indian monk seal

narwhal

white whales and narwhals

narwhals

baleen or whalebone whales

proboscis monkey

Australian sea lion

finless porpoise from Japan and Korea lion de mer d'Australie

marsouin de Chine

marsouin de l'Inde

baleine-à-bec de Biainville

baleine-à-bec de Gervais

baleine-à-bec de Gray

baleine-à-bec de NouvelleZélande

mésoplodon de Layard

baleine-à-bec de True

baleine-à-bec de Stejneger

éléphants de mer

éléphant de mer du nord

éléphant de mer du sud

phoques moines

phoque moine de la Méditerranée

phoque moine des îles Hawaii

phoque moine des Indes

occidentales

narval

monodontidés

narvals

baleines à fanons

nasique

marsouin du Japon

1. Pilleri, G., and M. Gihr, On the Taxonomy and Ecology of the Finless Black Porpoise, Neophocaena (Cetacea, Delphinidae), Mammalia 39, 657-673, 1975. 
135. Odobenidae

Odobenus divergens $=\mathrm{O}$. rosmarus

136. Odobenus rosmarus

137. Odobenus rosmarus divergens Pacific-Arctic walrus

138. Odobenus rosmarus laptevi

139. Odobenus rosmarus rosmarus

140. Odontoceti

141. Ommatophoca rossi

142. Orcaella brevirostris

Orcaella fluminalis $=\mathbf{O}$. brevirostris

Orcella $=$ Orcaella

143. Orcininae

144. Orcinus orca

Orcinus rectipinna $=0$. orca

Otaria byronia $=$ Otaria

flavescens

145. Otaria flavescens

Otaria ursina $=$ Callorhinus ursinus

146. Otariidae

147. Otariini

148. Paenungulata

Pagophilus $=$ Phoca

149. Peponocephala electra

150. Phoca

151. Phoca caspica

152. Phoca fasciata

153. Phoca groenlandica

154. Phoca groenlandica groenlandica

155. Phoca groenlandica oceanica

156. Phoca hispida

157. Phoca hispida botnica

158. Phoca hispida krasheninikovi

159. Phoca hispida ladogensis

160. Phoca hispida ochotensis

161. Phoca hispida saimensis

Phoca insularis $=P$. vitulina

Phoca kurilensis $=\mathrm{P}$. vitulina

Phoca largha $=$ Phoca vitulina largha

Phoca richardii $=\mathrm{P}$. vitulina

162. Phoca sibirica

Baikal seal odobénidés

morse

morse Pacifique

morse de la mer des Laptev

morse de l'Atlantique

odontocètes

phoque de Ross

Arcelle de l'Irraouad(d)i

pilot and killer whales

orcinés

killer whale

épaulard

South American sea lion

otarie australe

eared seals (or sealions and and fur seals)

sea-lions

near-ungulates

little blackfish

seals

Caspian seal

ribbon seal

harp seal

Newfoundland harp seal

White-Sea harp seal

ringed seal

Baltic ringed seal

Bering Sea ringed seal

Ladoga ringed seal

Gichiga ringed seal

Saimaa ringed seal

otaries (otariidés)

lions de mer

subongulés

phoques

phoque de la Caspienne

phoque à bandes

phoque du Groenland (F.)

phoque de Terre-Neuve

phoque de la mer Blanche

phoque marbré (F.)

phoque annelé (Can.)

phoque marbré de la Baltique

phoque marbré de la mer de Béring

phoque marbré du lac Ladoga phoque marbré de Giziga

phoque marbré du lac Saimaa

phoque annelé du Baikal 
163. Phoca vitulina

164. Phoca vitulina concolor

165. Phoca vitulina largha

166. Phoca vitulina mellonae

167. Phoca vitulina richardii

Phocaena dallii $=$ Phocaenoides dallii

168. Phocaena dioptrica

169. Phocaena phocoena

170. Phocaena phocoena relicta

171. Phocaena sinus

Phocaena spinipinnis

Phocaena truei $=$ Phocaenoides dallii

Phocaena vomerina $=\mathbf{P}$. phocoena

173. Phocaenidae

174. Phocaenoides dallii

Phocaenoides truei $=P$. dallii

175. Phocarctos hookeri

176. Phocidae

177. Phocinae

178. Phocoidea

Physeter catodon $=\mathbf{P}$. macrocephalus

179. Physeter macrocephalus

180. Physeteridae

181. Physeteroidea

182. Pinnipedia

183. Platanista gangetica

Platanista indi $=P$. minor

184. Platanista minor

185. Platanistidae

186. Platanistoidea

187. Pontoporia blainvillei

Prodelphinus $=$ Stenella

188. Pseudorca

189. Pseudorca crassidens

Pusa $=$ Phoca

Pusa hispida $=$ Phoca hispida

Pusa sibirica $=$ Phoca sibirica harbor seal

Atlantic harbor seal

Kamchatkan harbor seal

Ungava harbor seal

British Columbia harbor seal

spectacled porpoise

harbor porpoise

Black Sea harbor porpoise

Gulf of California porpoise

black porpoise

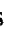

porpoises

Dall porpoise

New Zealand sea lion

true seals (earless seals)

northern seals

seals

sperm whale

sperm whales

sperm whales

pinnipeds (seals and their

allies in which the flippers

replace the feet)

Ganges susu

phoque veau-marin (F.)

phoque commun (Can.)

phoque veau-marin de

l'Atlantique

phoque veau-marin de

Kamchatka

phoque veau-marin de l'Ungava

phoque veau-marin de la

Colombie-Britannique

marsouin des lunettes

marsouin commun

marsouin commun de la mer

Noire

marsouin du Pacifique

marsouin spinnipenne

phocénidés

marsouin des estuaires

lion de mer de

Nouvelle-Zélande

phocidés

phoques du nord

phoques

cachalot à grosse tête

physétéridés

physétéridés

pinnipèdes

dauphin du Gange

Indus susu

gangetic dolphins

river dolphins

franciscana

lesser killer whales

false killer whale dauphin de l'Indus

platanistidés

dauphins d'eau douce

dauphin de la Plata

faux orques

pseudorque à dents épaisses 
Rhachianectes $=$ Eschrichtius

Rhachianectes glaucus $=$

Eschrichtus robustus

Rhytina $=$ Hydrodamalis

Rhytina stelleri $=$ Hydromalis gigas

Sibbaldus $=$ Balaenoptera

190. Sirenia

sea cows, dugongs, and

Sotalia borneensis $=$ Sousa chinensis

Sotalia brasiliensis $=\mathrm{S}$.

fluviatilis

Sotalia chinensis $=$ Sousa chinensis

191. Sotalia fluviatilis

192. Sotalia fluviatilis fuviatilis

193. Sotalia fluviatilis guianensis Sotalia gadamu $=$ Tursiops truncatus

Sotalia guianensis $=\mathrm{S}$.

fluviatilis guianensis

Sotalia lentiginosa $=$ Sousa chinensis

Sotalia pallida $=\mathbf{S}$. fluviatilis

Sotalia plumbea $=$ Sousa

chinensis

Sotalia sinensis $=$ Sousa chinensis

Sotalia teuszii $=$ Sousa teuszii

Sotalia tucuxi $=$ S. fluviatilis

Sousa borneensis $=S$. chinensis

South American tookashee Amazon River tookashee

Venezuela tookashee sotalie de l'Amérique du sud sotalie de l'Amazone sotalie du Venezuela
194. Sousa chinensis

Indo-Pacific humpback dolphin

sotalie de Chine

Sousa lentiginosa $=\mathrm{S}$.

chinensis

Sousa plumbea $=\mathrm{S}$. chinensis

Sousa queenslandensis $=\mathbf{S}$. chinensis

Sousa sinensis $=\mathbf{S}$. chinensis

195. Sousa teuszi

196. Stenella

Stenella alope $=\mathbf{S}$. longirostris

197. Stenella attenuata

198. Stenella attenuata graffmani

bridled dolphin

coastal bridled dolphin

Atlantic humpback dolphin

spotted dolphins

sotalie de Teusz

dauphin douteux manatees

siréniens

dauphins tachetés

Stenella caeruleoalba $=$ S. coeruleoalba 
199. Stenella coeruleoalba

Stenella dubia $=$ S. attenuata

Stenella euphrosyne $=\mathbf{S}$.

coeruleoalba

Stenella fraenata $=\mathbf{S}$. attenuata

Stenella frontalis $=\mathrm{S}$. attenuata

Stenella graffmani $=\mathbf{S}$.

attenata graffmani

200. Stenella longirostris

Stenella malayana $=$ Sousa

chinensis

Stenella mediterranea $=\mathbf{S}$.

attennata

Stenella microps $=\mathbf{S}$.

longirostris

Stenella permettensis $=\mathbf{S}$. plagiodon

201. Stenella plagiodon

Stenella roseiventris $=\mathrm{S}$.

longirostris

Stenella santonica $=\mathbf{S}$

coeruleoalba

Stenella styx $=\mathrm{S}$. coeruleoalba

202. Stenidae

203. Steno bredanensis

Steno rostratus $=$ Steno bredanensis

Stenodelphis = Pontoporia

Stenorhinchus $=$ Hydrurga

Stenorhynchus $=$ Hydrurga

Susu $=$ Platanista

204. Tasmacetus shepherdi

Shepherd's beaked whale

long-snouted dolphins

rough-toothed dolphin

sténidés

sténo rostré

tasmacète de Shepherd

205. Thalarctos. cf. Ursus maritimus (219)

206. Trichechidae

207. Trichechus inunguis

manatees

Trichechus latirostris $=\mathrm{T}$. latirostris

208. Trichechus manatus

Caribbean manate

209. Trichechus manatus latirostris Florida manatee

210. Trichechus manatus manatus

211. Trichechus senegalensis

213. Tursiops

South-American manatee

West African manatee

bottle-nosed dolphins

Tursiops abusalun $=T$. truncatus

214. Tursiops aduncus

Tursiops catalania $=T$ truncatus dauphin bleu (-blanc)

\section{lamantins}

lamantin d'Amérique du Sud

lamantin d'Amérique du Nord lamantin de Floride

lamantin de l'Amérique du Sud lamantin d'Afrique souffleurs 
Tursiops gadamu $=\mathrm{T}$.

truncatus

Tursiops gillii $=\mathrm{T}$. truncatus

Tursiops nesarnack $=\mathrm{T}$.

truncatus

215. Tursiops mangeanus

Tasmanian bottle-nosed dolphin

Tursiops nuuana (nuuanu) $=$ T. truncatus

216. Tursiops truncatus

Tursiops tursio $=\mathrm{T}$. truncatus

217. Ursidae

218. Ursus

219. Ursus maritimus

220. Zalophus californianus

221. Zalophus californianus japonicus

222. Zalophus californianus wollebaeki

Zalophus cinereus $=$

Neophoca cinerea

Zalophus japonicus $=\mathrm{Z}$

californianus japonicus

Zalophus lobatus $=$ Neophoca

cinerea

Zalophus wollebaeki $=\mathrm{Z}$.

californianus wollebaeki

223. Ziphiidae

224. Ziphius cavirostris bottle-nosed dolphin

bears

true bears

polar bear

California(n) sea lion

Japanese sea lion

Galapagos sea lion

\section{beaked whales}

goosebeak whale souffleur de Tasmanie

dauphin à gros nez

ursidés

ours

ours blanc

otarie de Californie

otarie du Japon

lion de mer de Galapagos

\section{English index}

bear, polar, 219

bears, 217

bears, polar, 205

bears, true, 218

beater: fully moulted harp-seal pup

bedlamer: immature harp seal

beluga, $\mathbf{5 0}$

beluga, Okhotsk Sea, 51

belukha $=$ beluga

blackfish $=$ whale, (longfin) pilot

blackfish, Atlantic = whale, (longfin) pilot

blackfish, little, 149

blackfish, many-toothed = blackfish, little

blackfish, melon-head = blackfish, little

blackfish, short-finned $=$ whale, shortfin pilot

bladdernose $=$ seal, hooded

blueback: seal, (young) hooded

bottle-head $=$ whale, pilot

bottle-nose $=$ whale, pilot

bottle-nose, white-sided $=$ dolphin, Atlantic whiteside

bouto = dolphin, Amazon

bowhead $=$ whale, bowhead ziphiidés

baleine-à-bec de Cuvier

buffeo negro $=$ tookashee, Amazon river cachalot $=$ whale, sperm

cachalot, lesser $=$ whale, pygmy sperm carnivores (flesh-eaters), 40

cetaceans, toothed $=$ whales, toothed chungungo, 103

cochito = porpoise, Gulf of California

cowfish $=$ whales, beaked (genus)

cowfish $=$ grampus, whitehead

crab eater $=$ seal, crab-eater

crested seals and sea elephants, 49

dolphin, Amazon, 84

dolphin, Amazonian = dolphin, Amazon

dolphin, Amazonian white $=$ tookashee, South American

dolphin, Arabian Sea saddleback, 56

dolphin, Atlantic spotted $=$ dolphin, spotted

dolphin, Atlantic humpback, 195

dolphin, Atlantic whiteside, 88

dolphin, black, 43

dolphin, blackchin, 90

dolphin, blue $=$ dolphin, striped

dolphin, blue-white $=$ dolphin, striped 
dolphin, Bornean $=$ dolphin, shortsnouted whitebelly

dolphin, Bory's, 54

dolphin, bottle-nose(d), 216

dolphin, bridled, 197; cf. also coastal, offshore, and Hawaiian bridled dolphins

dolphin, Cape $=$ dolphin, saddleback

dolphin, Ceylon $=$ dolphin, spinner

dolphin, Chilean $=$ dolphin, black

dolphin, Chinese $=$ dolphin, whiteflag

dolphin, Chinese river $=$ dolphin, whiteflag

dolphin, Chinese white $=$ dolphin, Indo-Pacific humpback

dolphin, coastal bridled, 198

dolphin, Commerson's $=$ dolphin, piebald

dolphin, common $=$ dolphin, saddleback

dolphin, cruciger $=$ dolphin, hourglass

dolphin, dusky, 93

dolphin, euphrosyne $=$ dolphin, striped

dolphin, Fitzroy's = dolphin, dusky

dolphin, Ganges = susu, Ganges

dolphin, gangetic $=$ susu, Ganges

dolphin, Geoffroy's = dolphin, Amazon

dolphin, Gill's bottle-nosed = dolphin, bottlenosed

dolphin, Graffman's = dolphin, bridled

dolphin, Gray's white-sided, 94

dolphin, Guiana = tookashee, South American

dolphin, Guianan river $=$ tookashee, South American

dolphin, Hawaiian bridled: subspecies of Stenella attenuata; correct scientific name has not been determined

dolphin, Heaviside's, 44

dolphin, Hector's = dolphin, pied

dolphin, hookfin $=$ dolphin, Pacific whitesided

dolphin, hourglass, 91

dolphin, Indian broad-beaked = blackfish, little

dolphin, Indo-Pacific humpback, 194

dolphin, Indus $=$ susu, Indus

dolphin, Irawadi $=$ dolphin, Irrawaddy

dolphin, Irrawaddy, 142

dolphin, Irrawaddy river $=$ dolphin, Irrawaddy

dolphin, Japanese northern rightwhale, 99

dolphin, La Plata = franciscana

dolphin, long-beaked $=$ dolphin, spinner

dolphin, Malay = dolphin, Indo-Pacific humpback

dolphin, Malaysian $=$ dolphin, Indo-Pacific humpback

dolphin, narrow-snouted $=$ dolphin, bridled

dolphin, northern rightwhale, 98

dolphin, offshore bridled: subspecies of Stenella

attenuata; correct scientific name has not been determined

dolphin, Pacific $=$ dolphin, saddleback

dolphin, Pacific bottle-nosed $=$ dolphin, bottle-nose(d)

dolphin, Pacific whitesided, 92

dolphin, pantropical spotted $=$ dolphin, bridled

dolphin, Peale's = dolphin, blackchin

dolphin, Peron's $=$ dolphin, southern rightwhale dolphin, piebald, 42 dolphin, pied, 45

dolphin, plumbeous $=$ dolphin, Indo-Ppacific humpback

dolphin, Red Sea bottle-nosed, 214

dolphin, Risso's = grampus, whitehead (according to Burton)

dolphin, rough-toothed, 203

dolphin, saddleback, 55

dolphin, shortsnouted whitebeily, 87

dolphin, small-headed $=$ dolphin, spinner

dolphin, South Atlantic dusky = dolphin, dusky

dolphin, southern rightwhale, 100

dolphin, speckled $=$ dolphin, Indo-Pacific humpback

dolphin, spinner, 200

dolphin, spotted, 201

dolphin, spotted $=$ dolphin, bridled

dolphin, spotted $=$ dolphin, narrow-snouted

dolphin, striped, 199

dolphin, Styz $=$ dolphin, striped

dolphin, Tasmanian bottlenosed, 215

dolphin, tonine $=$ dolphin, Heaviside's

dolphin, Torres Strait $=$ dolphin, spinner

dolphin, West African white $=$ dolphin, Atlantic humpback

dolphin, white $=$ dolphin, Indo-Pacific humpback

dolphin, whitebeak(ed), 89

dolphin, white-bellied = dolphin, black

dolphin, whiteflag, 96

dolphin, whitefront $=$ dolphin, pied

dolphin, white-sided $=$ dolphin, Atlantic whiteside

dolphins, Amazon, 85

dolphins, bottle-nosed, 213

dolphins, Commerson's, 41

dolphins, freshwater $=$ dolphins, river

dolphins, Ganges = dolphins, gangetic

dolphins, gangetic, 185

dolphins, long-snouted, 202

dolphins, right whale, 97

dolphins, river, 186

dolphins, spotted, 196

dolphins, true 53

dolphins, typical (as family) $=$ dolphins and porpoises

dolphins and porpoises, 52

dugongs, 76

elephant seals, cf. seals, elephant

finback, common $=$ whale, fin

finner $=$ whale, fin

fish, devil $=$ whale, grey

fish, white $=$ beluga

flesh-eaters $=$ carnivores

flipper, a bottle-nosed dolphin (of TV fame)

flipper, square $=$ seal, bearded

franciscana, 187

fur seal, Alaskan = fur seal, northern

fur seal, Antarctic, 7

fur seal, Antipodean, 4

fur seal, Australian = fur seal, Antipodean

fur seal, Cape = fur seal, South African

fur seal, Chilean = fur seal, Juan Fernandez 
fur seal, Falkland, 3

fur seal, Galapagos, 5

fur seal, giant, 9

fur seal, Guadalupe, 6

fur seal, Juan Fernandez, 8

fur seal, Kerguelen $=$ fur seal, Antarctic, or fur seal, subantarctic

fur seal, New Zealand = fur seal, Antipodean

fur seal, northern, 38

fur seal, Pribiloff Islands = fur seal, northern

fur seal, South African, 11

fur seal, South American, 2

fur seal, Subantarctic, 12

fur seal, Tasmanian = fur seal, Victorian

fur seal, Victorian, 10

fur seal, Western Australian = fur seal, Antipodean

fur seals, sea lions and = seals, eared

fur seals, southern, 1

grampus $=$ whale, killer

grampus = grampus, whitehead

grampus, gray $=$ grampus, whitehead

grampus, whitehead, 74

grey back $=$ whale, grey

grindhval $=$ whale, (longfin) pilot

hair-seal: any otarid the hair of which is too coarse to be called fur

harbor seal, cf. seal, harbor

hard head $=$ whale, grey

hippopotamus, 77

hippopotamus, pigmy, 47

horsehead $=$ seal, grey

humpback $=$ whale, humpback

ingotok: whale, young bowhead

jacobite $=$ dolphin, piebald

jar, common $=$ seal, (immature and adult) ringed

kairalik $=$ whale, bowhead

kairulik (Eskimo) : seal, harp

kasigiak $($ Eskimo $)=$ seal, harbor

killer $=$ whale, killer

killer, pygmy $=$ whale, pygmy killer

killers, false $=$ whales, lesser killer

killer whales, cf, whales, pilot and killer

lumbalumba $=$ dolphin, Irrawaddy

manatee, Amazon, 207

manatee, Caribbean, 208

manatee, Florida, 209

manatee, North American = manatee, Caribbean

manatee, South-American, 210

manatee, West African, 211

manatee, West Indian = manatee, Caribbean

manatees, 206

minke $=$ whale, minke

monkey, proboscis, 129

morse $=$ walrus

mussel digger $=$ whale, grey

namu $=$ whale, killer

nare whale $=$ narwhal

narwhal(e), 125

narwhals, 127

near-ungulates, 148 netsivak $=$ seal, $($ Eskimo fur $)$ hooded

nisack = porpoise, harbor

nordcaper $=$ whale, North Atlantic right

orca $=$ whale, killer

otter, Eurasian, 104

otter, marine = chungungo

otter, sea, cf. sea otter

Peh $\mathrm{Ch}^{\prime} \mathbf{i}=$ Pei c'hi

Pei c'hi = dolphin, whiteflag

pelloch $=$ porpoise, harbor

pinnipeds (seals and their allies in which flippers

replace true feet), 182

poggy: whale, young bowhead

pophead $=$ whale, (longfin) pilot

porpoise $($ New-Zealand $)=$ dolphin, pied

porpoise, bicolored $=$ porpoise, spectacled

porpoise, black, 172

porpoise, black finless $=$ porpoise, finless

porpoise, Black Sea harbor, 170

porpoise, Burmeister's = porpoise, black

porpoise, common $=$ porpoise, harbor

porpoise, Dall, 174

porpoise, Dall's harbor $=$ porpoise, Dall

porpoise, Dall's $=$ porpoise, Dall

porpoise, finless, 132

porpoise from China, finless, 131

porpoise from China and Japan, finless, 133

porpoise from Japan and Korea, finless, 134

porpoise, finless black $=$ porpoise, finless

porpoise, Gulf of California, 171

porpoise, harbor, 169

porpoise, Pacific harbor $=$ porpoise, harbor

porpoise, Peale's = dolphin, blackchin

porpoise, spectacled, 168

porpoise, True's = porpoise, Dall

porpoise, whiteflank $=$ porpoise, Dall

porpoise, 173

razoback $=$ whale, fin

right-whale dolphin, cf. dolphin

rip sack $=$ whale, grey

rorqual, common $=$ whale, fin

rorqual, great northern $=$ whale, blue

rorqual, least $=$ whale, minke

rorqual, lesser $=$ whale, minke

rorqual, Rudolphi's $=$ whale, sei

rorqual, Sibbald's = whale, blue

rorquals, 18

rorquals (or fin whales), 34

saddle-back = seal, harp

sea canary $=$ beluga

sea cow, great northern = sea cow, Steller's

sea cow, Steller's, 78

sea cow, dugongs, and manatees, 190

sea elephant $=$ seal, souther elephant

sea horse $=$ walrus

seal, Atlantic bearded, 62

seal, Atlantic harbor, 164

seal, Atlantic = seal, grey

seal, Baikal, 162

seal, Baltic ringed, 157

seal, banded = seal, ribbon

seal, bay $=$ seal, harbor 
seal, bearded, 61

seal, Bering Sea ringed, 158

seal, bladdernosed $=$ seal, hooded

seal, British Columbia harbor, 167

seal, Caribbean monk = seal, West Indian monk

seal, Caspian, 151

seal, common $=$ seal, harbor

seal, crab-eater, 101

seal, crab-eating $=$ seal, crab-eater

seal, crested $=$ seal, hooded

seal, fiord $=$ seal, ringed

seal, Gichiga ringed, 160

seal, Greenland = seal, harp

seal, grey, 75

seal, Groenland = seal, harp

seal, harbor, 163

seal, harp, 153

seal, Hawaiian monk, 123

seal, hooded, 48

seal, horsehead $=$ seal, grey

seal, Jamaica $=$ seal, West Indian monk

seal, jar $=$ seal, ringed

seal, Kamchatkan harbor, 165

seal, Ladoga ringed, 159

seal, Lake Ladoga ringed $=$ seal, Ladoga ringed

seal, Lake Saimaa ringed = seal, Saimaa ringed

seal, larga $=$ seal, spotted

seal. Laysan monk $=$ seal, Hawaiian monk

seal, leonard, 79

seal, (Mediterranean) monk, 122

seal, Newfoundland harp, 154

seal, northern elephant, 119

seal, North-sea ringed $=$ seal, Baltic ringed

seal, Okhotsk (Sea) ringed $=$ seal Gichiga ringed

seal, Pacific bearded, 63

seal, ribbon, 152

seal, ringed, 156

seal, Ross, 141

seal, saddleback = seal, harp

seal, Saimaa ringed, 161

seal, sand = seal, harbor

seal, southern elephant, 120

seal, spotted = seal, Kamchatkan harbor

seal, Tasmanian fur = fur seal, Victorian

seal, Ungava harbor, 166

seal, Weddell, 95

seal, Weddell's = seal, Weddell

seal, West Indian monk, 124

seal, White-Sea harp, 155

seals, 150

seals (superfamily), 178

seals and their allies $=$ pinnipeds

seals, eared (or sea-lions and fur seals), 146

seals, earless $=$ seals, (true)

seals, elephant, 118

seals, monk, 121

seals, northern, 177

seals, proboscis $=$ crested seals and sea elephants (subfamily)

seals, southern, 102

seals, true (earless seals), 176

sea leopard = seal, leopard sea lion, Auckland = sea lion, New Zealand

sea lion, Australian, 130

sea lion, black = sea lion, California

sea lion, California(n), 220

sea lion, Galapagos, 222

sea lion, Hooker's = sea lion, New Zealand

sea lion, Japanese, 221

sea lion, New Zealand, 175

sea lion, northern, 67

sea lion, South American, 145

sea lion, southern $=$ sea lion, South American

sea lion, Steller's = sea lion, northern

sea lions, 147 or 66

sea lions and fur seals = seals, eared

sea otter, 57

sea otter, California = sea otter, southern

sea otter, Kamchatka, 58

sea otter, northern, 59

sea otter, southern, 60

sea ox $=$ walrus

silver jar: (freshly moulted young) ringed seal

sishuk = susu, Ganges

sishumar = susu, Ganges

sisumara $=$ susu, Ganges

sletbag $=$ whale, right

sniffer $=$ porpoise, harbor

sulfurbottom (sulphurbottom) $=$ whale, blue

susu $=$ dolphins, gangetic

susu, Ganges, 183

susu, Indus, 184

tookashee $=$ tookashee, Amazon river

tookashee, Amazon River, 192

tookashee, South American, 191

tookashee, Venezuela, 193

tucuxi $=$ tookashee, Amazon river

ugjuk $($ Eskimo $)=$ seal, bearded

vaquita $=$ porpoise, Gulf of California

walrus, 136

walrus, Atlantic $=$ walrus, Atlantic-Arctic

walrus, Atlantic-Arctic, 139

walrus, Laptev Sea, 138

walrus, Pacific $=$ walrus, Pacific-Arctic

walrus, Pacific-Arctic, 137

walruses, 135

whale, Antarctic blue, 29

whale, Antillean beaked, 111

whale, archbeak, 109

whale, Arctic(right) $=$ whale, bowhead

whale, Arnoux's = whale, souther giant bottlenose

whale, Arnoux's beaked $=$ whale, southern giant botle-nose

whale, Atlantic pilot $=$ whale, longfin pilot

whale, Atlantic right $=$ whale, North Atlantic right

whale, Australian $=$ whale, pygmy right

whale, Baird's beaked $=$ whale, North Pacific giant bottle-nose

whale, baleen $=$ whale, whalebone or any of several mystacocetids of the genus Balaena

whale, beaked: any of several physeterids with a prolonged muzzle 
whale, Bering Sea beaked $=$ whale, sabertooth whale, Biscayan right $=$ whale, North Attlantic right

whale, Biscayan (right) $=$ whale, right whale, black right $=$ whale, North Atlantic right whale, Blainville's $=$ whale, densebeak whale, blue, 27

whale, bottle-nose $(d)=$ whale, North Atlantic bottle-nose

whale, bowhead, 16

whale, Bryde $=$ whale Bryde's

whale, Bryde's, 26

whale, bunch $=$ whale, humpback

whale, caaing $=$ whale, (longfin) pilot

whale, Caa'ing = whale, (longfin) pilot

whale, ca'ing $=$ whale, (longfin) pilot

whale, Californian grey $=$ whale, grey

whale, coalfish $=$ whale, Sei

whale, common killer $=$ whale, killer

whale, Cuvier's beaked $=$ whale, goosebeak

whale, Cuvier's $=$ whale, goosebeak

whale, deepcrest, 108

whale, densebeak, 110

whale, dwarf sperm, 86 a

whale, false killer, 189

whale, fin, 31

whale, finner $=$ whale, blue

whale, flathead bottle-nose, 82

whale, Gervais' beaked $=$ whale, Antillean beaked

whale, Gervais $=$ whale, Antillean beaked

whale, giant beaked $=$ whale, North Pacific giant bottle-nose

whale, ginkgo-tooth, 112

whale, goosebeak, 224

whale, goosebeaked $=$ whale, goosebeak

whale, Gray's (beaked) = whale, scamperdown

whale, great polar $=$ whale, bowhead

whale, Greenland = whale, bowhead

whale, Greenland right $=$ whale, bowhead

whale, grey, 65

whale, Gulf Stream beaked $=$ whale, Antillean beaked

whale, herring $=$ whale, fin

whale, hump $=$ whale, humpback

whale, humpback, 105

whale, hunchbacked $=$ whale, humpback

whale, ice baleen $=$ whale, North Atlantic right

whale, Indian pilot $=$ whale, shortfin pilot

whale, Indo-Pacific beaked, 83

whale, killer, 144

whale, Layard's $=$ whale, straptooth

whale, little piked $=$ whale, minke

whale, (longfin) pilot, 71

whale, minke, 19

whale, Moore's beaked $=$ whale, archbeak

whale, New Zealand beaked, 114

whale, North Atlantic blue, 30

whale, North Atlantic bottle-nose, 81

whale, North Atlantic minke, 20

whale, (North Atlantic) right, 15

whale, North cape $=$ whale, North Atlantic right whale, northern $=$ whale, bowhead

whale, northern bottle-nosed $=$ whale, North

Atlantic bottle-nose

whale, northern hemisphere fin, 33

whale, northern hemisphere sei, 24

whale, northern pilot $=$ whale, longfin pilot

whale, northern right $=$ whale, right

whale, North Pacific blue $=$ whale, North Atlantic blue

whale, North Pacific giant bottle-nose, 37

whale, North Pacific minke, 22

whale, North Sea beaked, 107

whale, Pacific grey $=$ whale, grey

whale, Pacific pilot, 73

whale, Pacific right $=$ whale, $($ North Atlantic) right

whale, piked $=$ whale, minke

whale, pikeheaded $=$ whale, minke

whale, pilot $=$ whale, (longfin) pilot

whale, pollack $=$ whale, sei

whale, porpoise $=$ whale, southern giant bottle-nose

whale, pot $=$ whale, sperm

whale, pygmy blue $=$ whale, southern Indian Ocean blue

whale, pygmy killer, 68

whale, pygmy right, 39

whale, pygmy sperm, 86

whale, right, 13

whale, Rudolphi's $=$ whale, sei

whale, sabertooth, 117

whale, scamperdown, 113; may also be one of several species of ziphiid of the genus Mesoplodon

whale, scrag $=$ whale, North Atlantic right

whale, sei, 23

whale, Shepherd's beaked, 204

whale, shortfin pilot, 70

whale, southern bottle-nosed $=$ whale, flathead bottle-nose

whale, southern giant bottle-nose, 36

whale, southern hemisphere fin, 32

whale, southern hemisphere longfin pilot, 72

whale, southern hemisphere minke, 21

whale, southern hemisphere sei, 25

whale, southern Indian Ocean blue, 28

whale, southern pilot $=$ whale, longfin pilot

whale, southern right, 14

whale, southern right $=$ whale, $($ North Atlantic) right

whale, Sowerby's (beaked) $=$ whale, North Sea beaked

whale, sperm, 179

whale, Stejneger's beaked $=$ whale, sabertooth

whale, straptooth, 115

whale, straptoothed $=$ whale, straptooth

whale, tropical beaked $=$ whale, densebeak

whale, True's beaked, 116

whale, whalebone: any whale of the suborder Mystacoceti

whale, wolfish $=$ whale, sei

whale, white $=$ beluga 
whales, dolphins, and porpoises, 46

whales, baleen or whalebone, 128

whales, beaked, 223 or 106

whales, bottle-nosed, 80

whales, bowhead (right whales), 17

whales, finback $=$ rorquals (family)

whales, fin $=$ rorquals

whales, giant bottle-nosed, 35

whales, grey, 64

whales, lesser killer, 188

\section{Français : index alphabétique}

baleine-à-bec commune (hypérodon du nord), 81 baleine-à-bec d'Arnoux = baleine d'Arnoux

baleine-à-bec de Baird = baleine-à-bec, grande baleine-à-bec de Blainville, 110

baleine-à-bec de Cuvier, 224

baleine-à-bec de Gervais, 111

baleine-à-bec de Gray, 113

baleine-à-bec de Longman, 83

baleine-à-bec de Moore, 109

baleine-à-bec de Nouvelle-Zélande, 114

baleine-à-bec de Nouvelle-Zélande = tasmacète de Shepherd

baleine-à-bec des oies $=$ baleine-à-bec de Cuvier baleine-à-bec de Sowerby, 107

baleine-à-bec de Stejneger, 117

baleine-à-bec de True, 116

baleine-à-bec d'oie $=$ baleine-à-bec de Cuvier

baleine-à-bec du sud $=$ hypérodon du sud

baleine-à-bec, grand, 37

baleine-à-bosse $=$ rorqual-à-bosse

baleine-à-dents en lanière $=$ mésoplodon de Layard

baleine au sarde $=$ baleine noire

baleine australe $=$ baleine noire

baleine blanche $=$ béluga (vrai)

baleine bleue $=$ rorqual bleu

baleine boréale $(\mathrm{F}$.$) ; baleine franche ( \mathrm{Can}$.), 16

baleine d'Arnoux, 36

baleine de Baird = baleine-à-bec, grande

baleine de Biscaye = baleine noire

baleine de Bryde = balénoptère boréal de Bryde

baleine de Gervais = baleine-à-bec de Gervais

baleine de Groenland = baleine boréale

baleine de Layard = mésoplondon de Layard

baleine des Basques = baleine noire

baleine de Siebold $=$ baleine noire

baleine de Sowerby = baleine-à-bec de Sowerby

baleine de True $=$ baleine-à-bec de True

baleine du bec des oies = baleine-à-bec de Cuvier

baleine du Groenland = baleine boréale

baleine du Pacifique = baleine noire

baleine franche $=$ baleine boréale

baleine franche naine $=$ baleine pygmée

baleine franche noire $=$ baleine noire

baleine grise (de Californie), 65

baleine jubarte $=$ rorqual-à-bosse

baleine naine $=$ baleine pygmée whales, pilot, 69

whales, pilot and killer, 143

whales, right $=$ whales, bowhead

whales, Sowerby's = whales, beaked

whales, sperm (superfamily), 181

whales, sperm, 180

whales, toothed, 140

whales, true $=$ whales, bowhead

whales, whalebone $\mathrm{cf}$. whales, baleen

white whales and narwhals, 126

whitecoat: harp seal pup of (young) ringed seal

baleine noire, 13

baleine noire de l'Atlantique Nord, 15

baleine noire du Sud, 14

baleine pygmée, 39

baleine sarde $=$ baleine noire

baleines, 17

baleines-à-bec, 80

baleines-à-bec $=$ ziphiidés

baleines-à-dents $=$ odontocètes

baleines-à-fanons, 128

baleines à gros bec $=$ ziphiidés

baleines blanches $=$ monodontidés

baleines grises $=$ eschrichtiidés

baleines noires, 35

balénidés $=$ baleines

balénoptère boreal $=$ rorqual boréal

balénoptère boréal bleu $=$ rorqual bleu

balénoptère boréal de Bryde, 26

balénoptère de Bryde $=$ balénoptère boréal de Bryde

balénoptère de Sibbald $=$ rorqual bleu

balénoptères $=$ rorquals

balénoptèridés $=$ rorquals

béluga (vrai, 50

béluga de la mer d'Okhotsk, 51

cachalot $=$ cachalot à grosse tête

cachalot à grosse tête, 179

cachalot macrocéphale = cachalot à grosse tête

cachalot nain $=$ cachalot pygmée

cachalot pygmée, 86

cachalots $=$ physétéridés

callorhine de l'Alaska = otarie à fourrure du nord

callorhine des îles Pribilof $=$ otarie à fourrure du nord

carnivores, 40

céphalorhynques $=$ dauphins de Commerson

cétacés, 46

cochon de mer $=$ marsouin commun

cystophorinés, 49

dauphin-à-bec blanc, 89

dauphin-à-bec de Peale, 90

dauphin-à-dents obliques $=$ dauphin-à-flancs blancs du Pacifique

dauphin-à-dents rudes $=$ sténo rostré

dauphin-à-dos lisse, 98

dauphin-à-dos lisse du Japon, 99

dauphin-à-flancs blancs, 88 
dauphin-à-flancs blancs du Pacifique, 92 dauphin à grand nez $=$ dauphin à gros nez dauphin à gros nez, 216 dauphin-à-nez blanc $=$ dauphin-à-bec blanc dauphin à rostre blanc $=$ dauphin-à-bec blanc dauphin blanc $=$ béluga (vrai) dauphin blanc de Chine $=$ sotalie de Chine dauphin bleu (-blanc), 199 dauphin bleu-blanc $=$ dauphin bleu dauphin commun, 55 dauphin commun de la mer d'Oman, 56 dauphin d'eau douce de Chine, 96 dauphin de Bornéo, 87 dauphin de Commerson, 42 dauphin de Fitzroy, 93 dauphin de Gill = dauphin à gros nez dauphin de Heaviside, 44 dauphin de Hector, 45 dauphin de la Plata, 187 dauphin de l'Indus, 184 dauphin de l'Irraouad(d)i = orcelle de l'Irraouaddi

dauphin de Malaisie $=$ sotalie de Chine dauphin de Peron, 100

dauphin de Risso = dauphin gris dauphin de Téthys $=$ dauphin bleu (-blanc) dauphin de Thétis = dauphin bleu (-blanc) dauphin des anciens $=$ dauphin commun dauphin douteux, 197

dauphin du Gange, 183

dauphin du nord $=$ dauphin à dos lisse dauphin gris, 74

dauphin noir, 43

dauphin pie $=$ dauphin de Commerson dauphin pilote $=$ globicéphale noir de l'Atlantique (= grinde)

dauphin rayé $=$ dauphin bleu (-blanc)

dauphin souffleur $=$ dauphin à gros nez

dauphin tacheté $=$ dauphin douteux

dauphins à long bec $=$ sténidés

dauphins-baleines francs, 97

dauphins d'eau douce, 186

dauphins de Commerson, 41

dauphins du Gange = platanistidés

dauphins pilotes $=$ globicephales

dauphins tachetés, 196

dauphins typiques $=$ delpinidés

dauphins vrais, 53

delphinidés (dauphins), 52

dogling $=$ baleine-à-bec commune

dugongidés, 76

éléphant de mer du nord, 119

éléphant de mer du sud, 120

éléphants de mer, 118

épaulard, 144

épaulard à tête ronde $=$ globicéphale noir de l'Atlantique

éschrichtiidés, 64

faux épaulard = pseudorque à dents épaisses

faux orque $=$ pseudorque à dents épaisses

faux orques, 188

gibbar $=$ rorqual-à-bosse globicéphale $=$ globicéphale noir de l'Atlantique globi(o)céphale de l'Inde = globicéphale du

Pacifique

globicéphale de Siebold, 73

globicéphale d'Inde = globicéphale du Pacifique globicéphale du Pacifique, 70

globi(o)céphale du sud = globicéphale noir de l'hémisphère sud

globicéphale grinde $=$ globicéphale noir de l'Atlantique

globi $(o)$ céphale indien $=$ globicéphale $\mathrm{du}$ Pacifique

globi(o)céphale noir $=$ globicéphale noir de l'Atlantique

globicéphale noir de l'Atlantique (C.); grinde (F.) 72

globicéphale noir de l'hémisphère sud, 72

globicéphales, 69

grand dauphin $=$ dauphin à gros nez

grande baleine bleue $=$ rorqual bleu

grinde $=$ globicéphale noir de l'Atlantique

hippopotame, 77

hippopotame nain, 47

hypérodon $=$ baleine-à-bec commune

hypérodon du nord = baleine-à-bec commune

hypérodon du sud, 82

hypérodons $=$ baleines-à-bec

inia de Geoffroy = inie de Geoffroy

inie de Geoffroy, 84

iniidés, 85

jubarte $=$ rorqual à bosse

lagénorhynque à bec blanc = dauphin-à-bec blanc lagénorhynque à bec pointu = dauphin à flancs blancs

lagénorhynque à flancs blancs = dauphin à flancs blancs

lagénorhynque à rostre blanc = dauphin-à-bec blanc

lagénorhynque de Gill = dauphin-à-bec blanc

lamantin d'Afrique, 211

lamantin d'Afrique occidentale = lamantin d'Afrique

lamantin d'Amérique du Nord, 208

lamantin d'Amérique du Sud, 207

lamantin de Floride, 209

lamantin de l'Amazonie = lamantin d'Amérique du Sud

lamantin de l'Amérique du Sud, 210

lamantins, 206

léopard de mer = phoque léopard

léopard des mers = phoque léopard

lion de mer $=$ otarie de Steller

lion de mer australien $=$ lion de mer d'Australie

lion de mer californien = otarie de Californie

lion de mer d'Australie, 130

lion de mer de Californie $=$ otarie de Californie

lion de mer de Galapagos, 222

lion de mer de Nouvelle-Zélande, 175

lion de mer du nord Pacifique $=$ otarie de Steller

lion marin cendre $=$ lion de mer d'Australie

lion marin de Patagonie = otarie australe

lions de mer (genus), 66; (family group), 147 
lissodelphininés = dauphins-baleines francs loup marin d'esprit $=$ phoque commun loutre de mer, 57

loutre de mer australe, 60

loutre de mer de Kamtchatka, 58

loutre de mer septentrionale, 59

loutre eurasienne, 104

marsouin $=$ dauphin commun

marsouin à lunettes = marsouin des lunettes

marsouin blanc $=$ béluga

marsouin commun, 169

marsouin commun de la mer Noire, 170

marsouin de Burmeister $=$ marsouin spinnipenne marsouin de Chine, 131

marsouin de Dall $=$ marsouin des estuaires

marsouin de l'Inde, 132

marsouin des estuaires, 174

marsouin des lunettes, 168

marsouin de True $=$ marsouin des estuaires

marsouin d'Orbigny $=$ dauphin gris

marsouin du Pacifique, 171

marsouin gris $=$ dauphin gris

marsouin spinnipenne, 172

marsouins $=$ phocidés

mégaptère $=$ baleine-à-bosse

mégaptère $=$ rorqual-à-bosse

mégaptère boops $=$ rorqual-à-bosse

mésoplodon de Gervais = baleine-à-bec de Gervais

mésoplodon de Layard, 115

mésoplodon de Sowerby $=$ baleine-à-bec de Sowerby

mésoplodon de True $=$ baleine-à-bec de True

mésoplodons, 106

monodontidés, 126

morse, 136

morse de la mer des Laptev, 138

morse de l'Atlantique, 139

morse du Pacifique $=$ morse Pacifique

morse Pacifique, 137

morses $=$ odobénidés

mystacocètes $=$ baleines-à-fanons

mysticètes $=$ baleines-à-fanons

narval, 125

narvals, 127

nasique, 129

nésarnak = dauphin à gros nez

nonodon $=$ narval

odobénidés, 135

odontocètes, 140

orcelle de l'Irraouad(d)i, 142

orcinés, 143

orcinus = épaulard

orque = épaulard

orque épaulard = épaulard

orque gladiateur $=$ épaulard

orque naine, 68

otarie à crinière $=$ otarie australe

otarie à crinière d'Amérique du Sud = otarie australe

otarie à fourrure australienne $=$ otarie du sud de l'Australie otarie à fourrure d'Amérique du Sud = otarie à fourrure australe

otarie à fourrure de Guadalupe = otarie de Chilé otarie à fourrure de Nouvelle-Zélande $=$ otarie d'Australie

otarie à fourrure des îles Kerguelen, 12

otarie à fourrure australe, 2

otarie à fourrure d'Afrique du Sud, 11

otarie à fourrure d'Australie = otarie d'Australie otarie à fourrure de Tasmanie = otarie à fourrure d'Afrique du Sud

otarie à fourrure de Townsend, 6

otarie à fourrure du nord, 38

otarie à fourrure géante, 9

otarie australe, 145

otarie d'Australie, 4

otarie de Californie, 220

otarie de Chilé, 8

otarie de Galapagos, 5

otarie de Kerguelen, 7

otarie de Nouvelle-Zélande = lion de mer de

Nouvelle-Zélande

otarie des Fakland, 3

otarie des îles Kerguelen = otarie de Kerguelen

otarie de Steller, 67

otarie de Townsend $=$ otarie à fourrure de

Townsend

otarie du Japon, 221

otarie du sud de l'Australie, 10

otaries (otariidés), 146

otaries à fourrure $=$ otaries

otariidés $=$ otaries

otaries, morses et phoques $=$ pinnipèdes (ordre)

ours, 218

ursidés $=$ ours

ours blanc, 219

ours marins $=$ otaries

petit épaulard $=$ pseudorque à dents épaisses

phocénidés, 173

phocidés, 176

phoque à bandes, 152

phoque à barbe (F.), 61

phoque à capuchon, 48

phoque à crête $=$ phoque à capuchon

phoque annelé $=$ phoque marbré

phoque annelé du Baikal, 162

phoque à rubans = phoque à bandes

phoque à selle $=$ phoque du Groenland

phoque à trompe = éléphant de mer du sud

phoque barbu $=$ phoque à barbe

phoque barbu de l'Atlantique, 62

phoque barbu du Pacifique, 63

phoque chien de mer $=$ phoque veau-marin

phoque commun $=$ phoque veau-marin

phoque crabier, 101

phoque de la Caspienne, 151

phoque de la mer Blanche, 155

phoque de la mer Caspienne $=$ phoque de la Caspienne

phoque de Ross, 141

phoque de Sibérie $=$ phoque annelé du Baikal

phoque de Terre-Neuve, 154 
phoque de Weddell, 95

phoque du Baikal = phoque annelé du Baikal

phoque du Groenland (F), 153

phoque gris, 75

phoque léopard, 79

phoque marbré (F.); phoque annelé (Can.), 156

phoque marbré de Giziga, 160

phoque marbré de la Baltique, 157

phoque marbré de la mer de Béring, 158

phoque marbré du lac Ladoga, 159

phoque marbré du lac Saimaa, 161

phoque moine de Hawaii $=$ phoque moine des îles Hawaii

phoque moine de la Méditerranée, 122

phoque moine de Laysan $=$ phoque moine des îles Hawaii

phoque moine des Caraibes $=$ phoque moine des Indes occidentales

phoque moine des îles Hawaii, 123

phoque moine des Indes occidentales, 124

phoque moine méditerranéen $=$ phoque moine de la Méditerranée

phoque veau-marin (F.); phoque commun (Can.), 163

phoque veau-marin de Kamtchatka, 165

phoque veau-marin de la Colombie-Britannique, 167

phoque veau-marin de l'Atlantique, 164

phoque veau-marin de l'Ungava, 166

phoques (genus), 150; (family), 178

phoques à trompe $=$ cystophorinés

phoques du nord, 177

phoques du sud, 102

phoques moines, 121

physétéridés (family), 180 ; (superfamily), 181

pinnipèdes, 182

plataniste $=$ dauphin du Gange

platanistidés, 185

platanistoidés $=$ dauphins d'eau douce

pourceau de mer $=$ marsouin commun

pourcil = marsouin commun

pseudorque à dents épaisses, 189

pseudorques $=$ faux orques

rhytine de Steller, 78

rorqual à bec $=$ rorqual, petit

rorqual à bosse (Can.), 105

rorqual, petit, 19

rorqual à museau pointu = rorqual, petit

rorqual à rostre $=$ rorqual, petit

rorqual bleu, 27

rorqual bleu de l'Antarctique, 29

rorqual bleu, petit, 28

rorqual bleu de l'Atlantique Nord, 30

rorqual boréal, 23 rorqual boréal de l'hémisphère nord, 24

rorqual boréal de l'hémisphère sud, 25

rorqual commun, 31

rorqual commun de l'hémisphère nord, 33

rorqual commun de l'hémisphère sud, 32

rorqual de Bryde = balénoptère boréal de Bryde rorqual de l'Atlantique Nord, petit, 20

rorqual de l'hémisphère sud, petit, 21

rorqual de Rudolphi $=$ rorqual boréal

rorqual de Sibbald $=$ rorqual bleu

rorqual du nord $=$ rorqual boréal

rorqual du Pacifique Nord, petit, 22

rorqual sei $=$ rorqual boréal

rorquals (genus), 18 ; (family), 34

sarde $=$ baleine noire

siréniens, 190

sotalie de Chine, 194

sotalie de la Guyane = sotalie de l'Amérique du Sud

sotalie de l'Amazone, 192

sotalie de l'Amazone = sotalie de l'Amérique du

Sud

sotalie de l'Amérique du Sud, 191

sotalie de Teusz, 195

sotalie du Cameroun $=$ sotalie de Teusz

sotalie du Venezuela, 193

souffleur de Gill = dauphin à gros nez

souffleur de la Mer Rouge = tursiops de la Mer Rouge

souffleur de Tasmanie, 215

souffleur du Pacifique $=$ dauphin à gros nez

souffleur nesarnack $=$ dauphin à gros nez

souffleurs, 213

stellère $=$ rhytine de Steller

sténidés, 202

sténo rostré, 203

subongulés, 148

tasmacète de Shepherd, 204

tête de cheval $=$ phoque gris

trichéchides = lamantins

tursion de la Mer Rouge = tursiops du Mer Rouge

tursion souffleur $=$ dauphin à gros nez

tursions $=$ souffleurs

tursiops $=$ souffleurs

tursiops de la Mer Rouge, 214

tursiops tronqué $=$ dauphin à gros nez

ursidés, 217

vache marine $=$ morse

veau marin $=$ phoque veau marin

ziphiidés, 223

\section{BIBLIOGRAPHIE}

ANDERSON, S. and J. K. JONES, jr. (editors), 1967, Recent Mammals of the World: A Synopsis of Families, Ronald Press, New York, N.Y.

BURT, W. H. (editor), 1972, Antarctic Pinnipedia, American Geophysical Union.

CHAPSKII, K. K. and V. E. Sokolov (editors), 1973, Morphology and Ecology of Marine Mammals, J. Wiley, New York, N.Y. 
GASKIN, D. E., 1970, Whales, Dolphins and Seals, St. Martin's Press, New York, N.Y.

HERSHKOVITZ, P., 1966, Catalog of Living Whales, U.S. Natl. Mus. Bull. No. 246.

International Union for Conservation of Nature and Natural Resources, 1972, Red Data Book, Vol. 1, Mammalia, Morges, Suisse.

International Whaling Commission, Subcommittee on Smaller Cetaceans, Montreal, Canada, April I-II, 1974. J. Fish. Res. Board Canada 32, 889-983.

KING, J. E., 1964, Seals of the World, Br. Mus. (Nat. Hist), London.

KLEINENBERG, S. E., 1958, On the Origin of Cetacea, Proc. 15th Int. Congr. Zool. 445-447.

MATTHES, H. W., 1962, «Verbreitung der Saeugetiere in der Vorzeit», In: Handbuch der Zoologie, Band 8, Lief. 28, 11(1), 1-198. Walter de Gruyter \& Co., Berlin.

MITCHELL, E. and R. H. Tedford, 1973, The Enaliarctinae, a New Group of Extinct Aquatic Carnivora and $a$ Consideration of the Origin of the Otariidae, Bull. Am. Mus. Nat. Hist. $151,201-284$.

PIVETEAU, J. (editor), 1958, Traité de paléontologie, Tome 6, Vol. 2, Masson, Paris. 1961, Traité de paléontologie, Tome 6, Vol. 1, Masson, Paris

REINHART, R. H., 1959, A Review of the Sirenia and Desmostylia, Univ. Calif. Publ. Geol. Sci. $36,1-146$.

ROMER, A. S., 1966, Vertebrate Paleontology, Third Edition, Univ. Chicago Press, Chicago, Ill.

SCHEFFER, V. B., 1958, Seals, Sea Lions, and Walruses: A review of the Pinnipedia, Stanford Univ. Press, Stanford, Calif. 